\title{
Correction to: Novel Severe Acute Respiratory Syndrome Coronavirus 2 (SARS-CoV2) and Other Coronaviruses: A Genome-wide Comparative Annotation and Analysis
}

\author{
Mohammed Tarique ${ }^{1}$ (D) Shaban Ahmad ${ }^{2} \cdot$ Arshi Malik $^{3} \cdot$ Irfan Ahmad $^{4,5} \cdot$ Mohd Saeed $^{6} \cdot$ Ahmad Almatroudi $^{7}$. \\ Talal Qadah $^{8} \cdot$ Manal Abdulaziz Murad $^{9} \cdot$ Mutaib Mashraqi $^{10} \cdot$ Qamre Alam $^{11} \cdot$ Yousef Al-Saleh $^{12,13,14}$
}

Published online: 9 December 2021

(c) Springer Science+Business Media, LLC, part of Springer Nature 2021

\section{Correction to: \\ Molecular and Cellular Biochemistry \\ (2021) 476:2203-2217 \\ https://doi.org/10.1007/s11010-020-04027-8}

In the original publication of the article, funding text in the acknowledgement section was incorrectly published. The correct Acknowledgements section is provided in this correction.

Acknowledgements The authors acknowledge with much obliged for Almanac Life Science India Pvt. Ltd. for data

The original article can be found online at https://doi.org/10.1007/ s11010-020-04027-8.

Mohammed Tarique

tariqueaiims@gmail.com

1 Center for Interdisciplinary Research in Basic Sciences, Jamia Millia Islamia, Jamia Nagar, New Delhi 110025, India

2 Department of Computer Sciences, Jamia Millia Islamia, New Delhi 110025, India

3 Department of Clinical Biochemistry, College of Medicine, King Khalid University, Abha, Saudi Arabia

4 Department of Clinical Laboratory Science, College of Applied Medical Sciences, King Khalid University, Abha, Saudi Arabia

5 Research Centre for Advanced Materials Science, King Khalid University, Abha, Saudi Arabia

6 Department of Biology College of Sciences, University of Hail, Hail, Saudi Arabia

7 Department of Medical Laboratories, College of Applied Medical Sciences, Qassim University, Buraydah 51431, Saudi Arabia

8 Department of Medical Laboratory Technology, Faculty of Applied Medical Sciences, King Abdulaziz University, P.O. Box 80324, Jeddah Postcode 21589, Saudi Arabia analysis and valuable inputs. This work is funded by the Deanship of Scientific Research at King Khalid University Abha, Saudi Arabia through the Research group Project under the grant number (R.G.P. 01/48/42).

Publisher's Note Springer Nature remains neutral with regard to jurisdictional claims in published maps and institutional affiliations.
9 Department Family Medicine, Faculty of Medicine, King Abdulaziz University, Jeddah, Saudi Arabia

10 Department of Clinical Laboratory Sciences, College of Applied Medical Science, Najran University, Najran, Saudi Arabia

11 Medical Genomics Research Department, King Abdullah International Medical Research Center (KAIMRC), King Saud Bin Abdulaziz University for Health Sciences, King Abdulaziz Medical City, Ministry of National Guard Health Affairs, Riyadh 11426, Saudi Arabia

12 College of Medicine, King Saud Bin Abdulaziz University for Health Sciences, Riyadh 22490, Saudi Arabia

13 King Abdullah International Medical Research Center, Riyadh 11481, Saudi Arabia

14 Department of Medicine, King Abdulaziz Medical City, Ministry of National Guard-Health Affairs, Riyadh 14611, Saudi Arabia 